\title{
Necesidades Terapéuticas en Agresores de Pareja según su Perfil Diferencial
}

\section{Therapeutic Needs of Partner-Violent Men According to their Differential Profile}

\author{
Ismael Loinaz \\ Universidad del País Vasco - FPI Gobierno Vasco
}

\author{
Enrique Echeburúa \\ Universidad del País Vasco - CIBERSAM
}

\begin{abstract}
Resumen. La violencia contra la pareja es un problema que se debe abordar desde diferentes perspectivas. El tratamiento adecuado de los agresores debe ser uno de los objetivos prioritarios en su prevención. Tratar no implica eximir al agresor de su responsabilidad y es esencial para evitar futuras agresiones. En nuestro entorno se ha analizado en los últimos años la existencia de distintos perfiles de agresores, así como la eficacia de determinadas intervenciones. Los distintos tipos de agresores encontrados han permitido describir sus necesidades terapéuticas diferenciales y conocer qué aspectos deben ser modificados mediante las terapias. El presente trabajo pretende resumir los hallazgos sobre la clasificación de los agresores y sobre el diseño de programas adaptados a sus necesidades terapéuticas.
\end{abstract}

Palabras clave: violencia contra la pareja, tratamiento psicológico, tipologías de agresores.

\begin{abstract}
Intimate partner violence is a problem that must be approached from different perspectives. The adequate treatment of offenders should be a priority in its prevention. Treatment does not imply to relieve the offender of responsibility and is essential to prevent future attacks. In our cultural setting the existence of different offender profiles and the effectiveness of certain interventions have been analyzed in recent years. The different types of partner-violent men found in our research has allowed to describe their differential therapeutic needs and to know what aspects should be modified by therapy. This paper aims to summarize the latest evidence on the classification of partner-violent men and on the design of programs adapted to their therapeutic needs.
\end{abstract}

Key words: intimate partner violence, psychological treatment, batterer typologies.

\section{Introducción}

Tratar o rehabilitar a un agresor implica modificar aquellas características del sujeto que lo impulsan a agredir. No significa que el agresor sea un enfermo o deje de ser responsable de sus actos (Echeburúa, Corral,

La correspondencia sobre este artículo puede dirigirse a Ismael Loinaz. Facultad de Psicología. Universidad del País Vasco. 20018 San Sebastián (Spain). E-mail: ismael.loinaz@gmail.com

Agradecimientos: parte del trabajo descrito ha sido posible gracias a la colaboración del Centro Penitenciario Brians-2 y la ayuda económica del Centro de Estudios Jurídicos y Formación Especializada (Barcelona). La colaboración del equipo terapéutico de Brians-2 ha sido imprescindible en el diseño y la aplicación de los programas de tratamiento descritos (Alba García, Cristina Guillot, Ferran Domènech, Isabel Jodar, Juan Carlos Gil, Juanka Duran y Montse Solsona). 
Fernández-Montalvo y Amor, 2004), aunque en contadas ocasiones esto pueda ocurrir. Aceptar la existencia de determinados déficits o alteraciones conductuales, así como otras variables implicadas en los episodios violentos, sólo es el primer paso hacia la rehabilitación del sujeto y hacia el cese de la violencia contra su pareja o contra una futura pareja.

Existen distintas variables implicadas en la violencia contra la pareja (VCP). Algunos factores de riesgo son estáticos (la edad del primer delito -ser joven-, la violencia en la familia de origen, la conducta antisocial, la agresión previa a la pareja o a otras parejas y los trastornos mentales severos) y otros son dinámicos (como la ira, las distorsiones cognitivas y las actitudes machistas o favorables a la violencia, el consumo de drogas, la dependencia emocional y algunos problemas psicológicos). La motivación delictiva está integrada, en buena medida, por factores dinámicos modificables (Redondo, 2008). Por tanto, el tratamiento de los agresores de pareja debe centrarse en este tipo de factores, que mediante su modificación pueden alterar la motivación del sujeto para agredir a su pareja.

Así pues, el análisis exhaustivo de estos factores en cada sujeto, variables de un caso a otro, es el punto de partida imprescindible para diseñar programas de tratamiento efectivos. El objetivo de un tratamiento eficaz debe ser conseguir un cambio conductual estable en el tiempo. Se sabe que las recaídas son más frecuentes en los primeros meses posteriores al tratamiento y que las estrategias aprendidas suelen afianzarse en torno a los dos años (Echeburúa, 2004). Por otro lado, parece ser que las recaídas dependen más de características personales del agresor que del tipo de tratamiento recibido, lo que concede una gran relevancia al estudio de las tipologías y a la adecuación de los tratamientos a los diferentes subtipos. Éste es, precisamente, el objetivo central del presente estudio.

\section{Tipologías de agresores}

\section{Modelos internacionales y nuestra experiencia}

Distintos estudios internacionales han analizado la existencia de tipologías de agresores de pareja y han coincidido principalmente en la existencia de tres tipos de agresores: normalizados o limitados a la pareja, borderline/disfóricos o patológicos y violentos en general/antisociales (ver Amor, Echeburúa y Loinaz, 2009; Loinaz, 2009).

En nuestro entorno se ha estudiado el perfil diferencial de los agresores en distintos estudios (Echeburúa y Fernández-Montalvo, 2007; Fernández-Montalvo y Echeburúa, 2008; Fernández-Montalvo, Echeburúa y Amor, 2005) y recientemente se ha comenzado a aplicar la aproximación tipológica en entornos penitenciarios (Loinaz, 2009, 2010; Loinaz, Echeburúa y Torrubia, 2010; Loinaz, Ortiz-Tallo, Sánchez y Ferragut, 2011). Hasta la fecha, los resultados han permitido avalar parcialmente las propuestas internacionales al establecer dos grupos de agresores diferenciados:

1. Los sujetos "violentos con la pareja/estables emocionalmente/integrados socialmente", equivalentes al tipo normalizado, se caracterizan por una violencia limitada a la pareja, menor número de distorsiones cognitivas, menor abuso de drogas, menor presencia de antecedentes penales, mayor autoestima, mayor control de la ira, poca presencia de síndromes clínicos y estilos de personalidad compulsivos, narcisistas e histriónicos. Con un estilo de apego predominantemente seguro, tienen mayor empatía y muestran mayor deseabilidad social.

2. Los sujetos "violentos generalizados/poco estables emocionalmente/no integrados socialmente" se caracterizan por una violencia que se extiende más allá de la pareja y presentan antecedentes penales con mayor frecuencia. Estos sujetos están más afectados por síntomas clínicos, tienen más distorsiones cognitivas, dependen más del alcohol/drogas y muestran rasgos de personalidad antisocial, paranoide y negativista. Asimismo, son más impulsivos y se muestran menos empáticos que los sujetos del grupo anterior, con estilos de apego inseguro u hostil. 


\section{Implicaciones de las tipologías}

La tasa de abandonos en los programas de tratamiento de agresores de pareja oscila entre el $50 \%$ y el $75 \%$ (Daly y Pelowski, 2000) y la reincidencia en sujetos tratados varía del 20\% al 60\% (Gondolf y White, 2001; Klein y Tobin, 2008), pero ésta se puede duplicar en aquellos sujetos que no completan la terapia (Dutton, Bodnarchuk, Kropp, Hart y Ogloff, 1997; Gondolf, 2000). A su vez, el cambio psicológico producido tras el tratamiento y el cese de la violencia inicial no siempre implican cambios conductuales a largo plazo (Bowen, Gilchrist y Beech, 2008; Klein y Tobin, 2008).

Esta ineficacia parcial de los tratamientos ha sido achacada a la falta de consideración de la heterogeneidad de los agresores, así como a la aplicación de programas estandarizados que ignoran las necesidades terapéuticas de cada tipo de agresor (ver Loinaz, Torrubia, Echeburúa, Navarro y Fernández, 2009).

\section{Programas de tratamiento}

\section{Aspectos generales}

Existen distintas modalidades terapéuticas: individuales, grupales e incluso terapias de pareja. Esta última está recomendada únicamente en algunos casos concretos, como son los sujetos de bajo riesgo, alta motivación por el tratamiento, violencia leve y sin abuso de drogas (Stith, Rosen y McCollum, 2003; Stith, Rosen, McCollum y Thomsen, 2004). Las intervenciones grupales incrementan la probabilidad de que el agresor identifique sus pensamientos y conductas problemáticas a través de otros miembros del grupo (Johnson, 2007). Las intervenciones individuales han recibido menor atención, posiblemente debido a su mayor coste y la imposibilidad de aplicarlas en contextos judiciales. Pese a ello, es evidente que la atención que en ellas se puede prestar al paciente es superior (Echeburúa, Sarasúa, Zubizarreta y Corral, 2009), aunque se pierden determinados beneficios que ofrece la terapia grupal.

Pese a que no existen estudios exhaustivos que comparen distintas modalidades terapéuticas, los programas cognitivo-conductuales ofrecen los mejores resultados en la reducción de la reincidencia (Dutton, 2008; Johnson, 2007). Su objetivo es ayudar a que el sujeto reconozca los signos cognitivos, físicos y emocionales que preceden a su violencia y los pensamientos irracionales que la mantienen, utilizando técnicas como el tiempo fuera, relajación, monitorización conductual y entrenamiento en asertividad (Johnson, 2007; Russell, 2002). Como crítica, Dutton (2008) indica que poco se suele hacer sobre las fuentes de estrés, como el miedo al abandono o los celos, base motivacional de los comportamientos controladores de la pareja. En general, estos programas han demostrado buenos resultados respecto a la reducción de la agresión física, pero los estudios longitudinales demuestran la ineficacia de muchos de ellos.

\section{Estructura de los programas y protocolos de evaluación}

La mayoría de los programas de tratamiento comparten una estructura similar, ya sean de corte individual o grupal. Sobre esta estructura varía la extensión y el contenido concreto de las sesiones (en la tabla 1 se presenta la estructura del programa individual de Echeburúa et al., 2009). El número promedio de sesiones es de quince-veinte y la duración de las sesiones varía de 1 a 3 horas (ver Dutton, 2007, 2008; Dutton y Sonkin, 2003; Echeburúa y Corral, 1998; Echeburúa et al., 2009; Johnson, 2007; Russell, 2002). Por regla general, los agresores de bajo riesgo pueden beneficiarse de tratamientos centrados en el control de la impulsividad y la gestión de la ira (Cavanaugh y Gelles, 2005) y por sus características son los que menor intensidad y extensión requerirán. 
Tabla 1. Programa de tratamiento y diario de sesiones (adaptado de Echeburúa et al., 2009)

Contenido del tratamiento

a) Motivación ( $1^{\mathrm{a}}-4^{\mathrm{a}}$ sesión $)$

Aspectos motivacionales y empatía

b) Síntomas psicopatológicos $\left(5^{\mathrm{a}}-18^{\mathrm{a}}\right.$ sesión $)$

b.1) Primer nivel de intervención ( $5^{\mathrm{a}}-9^{\mathrm{a}}$ sesión)

- Ira descontrolada

- Ideas distorsionadas sobre los roles sexuales y sobre la violencia como forma de solución de problemas

- Ansiedad/estrés

b.2.) Segundo nivel de intervención $\left(10^{\mathrm{a}}\right.$ - $14^{\mathrm{a}}$ sesión $)$

- Déficits en la autoestima

- Celos patológicos

- Consumo abusivo de alcohol

b.3.) Tercer nivel de intervención ( $15^{\mathrm{a}}$ - $18^{\mathrm{a}}$ sesión)

- Déficits de asertividad y comunicación

- Déficits en la resolución de problemas

Déficits en las relaciones sexuales

c) Prevención de recaídas $\left(19^{\mathrm{a}}-20^{\mathrm{a}}\right.$ sesión)

- Situaciones de riesgo
Técnicas terapéuticas

Exposición a imágenes audiovisuales

Discusión racional y reestructuración cognitiva

Explicación del ciclo de la violencia y la escalada de ira

Suspensión temporal

Distracción cognitiva

Entrenamiento en autoinstrucciones

Educación sobre la igualdad de los sexos

Reestructuración cognitiva

Relajación

Reevaluación cognitiva

Reestructuración cognitiva y Saciación

Programa de bebida controlada

Entrenamiento en asertividad y habilidades de comunicación

Entrenamiento en solución de problemas

Educación sobre la sexualidad en la pareja

Entrenamiento en la aplicación de pautas de actuación urgentes

Tabla 2. Protocolo de evaluación con perspectiva tipológica

Instrumento

Entrevista estructurada

Inventario de pensamientos distorsionados (Echeburúa y Fernández-Montalvo, 1998; versión factorial de Ferrer, Bosch, Ramis, Torres y Navarro, 2006).

\section{Escala de autoestima de Rosenberg}

Inventario Clínico Multiaxial de Millon-III

(Adaptación española de Cardenal y Sánchez, 2007).

Inventario de Expresión de Ira Estado-Rasgo (STAXI-2)

(Miguel-Tobal, Casado, Cano-Vindel y Spielberger, 2001).

Escala de Impulsividad de Barratt (BIS-11) (versión española de Oquendo, Baca-García, Graver, Morales, Montalbán y Mann, 2001).

Conflict Tactics Scales-2 (CTS-2) de Straus, Hamby, Boney-McCoy y Sugarman (1996) (traducción de Loinaz, 2008).

Cuestionario de Apego Adulto (Malero y Cantero, 2008).

Índice de Reactividad Interpersonal (versión española de Mestre, Frías y Samper, 2004)

\section{Aspecto evaluado}

Variables sociodemográficas, descripción de la conducta violenta, historia evolutiva, etc. Pueden utilizarse versiones específicas que permitan evaluar la psicopatía (PCL-R o PCL-SV)

Distorsiones cognitivas sobre la mujer y sobre el uso de la violencia

Autoestima

Trastornos de la personalidad y síndromes clínicos (Eje I). Puede ser interpretado de forma dimensional.

Ira-estado e ira-rasgo, así como el control y la expresión de la misma.

Impulsividad

Modos de resolver conflictos en la pareja (negociación o agresión), así como la severidad de la violencia física. Puede ser una buena medida de cambio terapéutico.

Cuatro tipos de estilo afectivo

Cuatro dimensiones de empatía 
Respecto a los protocolos de evaluación, se recomienda actualizarlos y adaptarlos a cada situación según la disponibilidad, los objetivos y el contexto de aplicación. En la actualidad, en entornos penitenciarios, donde se está desarrollando la clasificación tipológica de los agresores de pareja, el protocolo recomendado es el descrito en la tabla 2. Sus principales objetivos son cubrir las distintas dimensiones de interés que permiten clasificar al agresor y ajustar el tratamiento, así como pronosticar la evolución del sujeto y su riesgo de reincidencia.

\section{Diseño de tratamientos basados en las tipologías}

Como se ha comentado, la violencia contra la pareja presenta determinados factores de riesgo que pueden ser modificados mediante el tratamiento psicológico. A su vez, existen distintos tipos de agresores que pueden ser identificados mediante protocolos de evaluación adecuados y que presentan características diferenciales, o distinta gravedad de síntomas, que los hacen merecedores de intervenciones específicas y adecuadas a sus necesidades. En definitiva, debemos ser capaces de comprender qué funciona, para qué agresores y en qué contextos. Posiblemente la elección más acertada sería el tratamiento individualizado de cada sujeto como caso único. Sin embargo, la magnitud del problema, la limitación de los recursos existentes y ciertos beneficios de las terapias grupales hacen que la asignación a grupos de tratamiento basados en el perfil del agresor sea una necesidad en nuestra sociedad actual.

Tabla 3. Características de cada subtipo y recomendaciones terapéuticas (adaptado de Loinaz, 2010)

\begin{tabular}{|c|c|c|c|}
\hline Variable & Normalizado & Límite/Patológico & Antisocial \\
\hline Extensión de la violencia & Limitada a la pareja & Predominante con la pareja & Frecuente agresión a desconocidos \\
\hline Tipo de violencia & Poco frecuente. Gravedad variable & Predominio de la violencia psicológica & Predominio de la agresión física \\
\hline Versatilidad delictiva & Sin antecedentes & No específica & Antecedentes frecuentes y diversos \\
\hline Estilo de apego & $\begin{array}{l}\text { Seguro: flexibles, se adaptan en las } \\
\text { relaciones y no necesitan manipular. } \\
\text { Expresan sus necesidades, habitual- } \\
\text { mente sin recurrir a la violencia. } \\
\text { Muestran mayor empatía } \\
\text { (algunos presentan un estilo } \\
\text { preocupado) }\end{array}$ & $\begin{array}{l}\text { Preocupado: menos distantes en las } \\
\text { discusiones. El abandono de la pareja } \\
\text { es el mejor predictor de su violencia. } \\
\text { Son celosos, dependientes y con abuso } \\
\text { psicológico } \\
\text { Temeroso: ansiosos e irritables ante el } \\
\text { abandono; hipersensibles al rechazo }\end{array}$ & $\begin{array}{l}\text { Rechazante: controladores y distantes. } \\
\text { El mayor precursor de su agresión es } \\
\text { la defensividad de la pareja. Muestran } \\
\text { poco interés o poca capacidad de reco- } \\
\text { nocer las cogniciones y emociones de } \\
\text { los demás }\end{array}$ \\
\hline Dependencia emocional & Moderada & Alta & Baja \\
\hline Consumo de drogas & $\begin{array}{l}\text { Similar a la población general. } \\
\text { Negación de la violencia y del consu- } \\
\text { mo de drogas. Posible relación entre el } \\
\text { consumo y la agresión, pero sin } \\
\text { dependencia }\end{array}$ & $\begin{array}{l}\text { Moderado/Alto. Relacionado con el } \\
\text { malestar afectivo. Recurren a las dro- } \\
\text { gas para calmar la ansiedad }\end{array}$ & $\begin{array}{l}\text { Alto (policonsumo). Patrón relaciona- } \\
\text { do con la búsqueda de sensaciones, } \\
\text { gratificación inmediata y hábitos } \\
\text { delictivos }\end{array}$ \\
\hline Ira/Hostilidad & $\begin{array}{c}\text { Tendencia a acumular ira hasta } \\
\text { explotar }\end{array}$ & Ira reactiva frente al abandono & $\begin{array}{l}\text { Expresión hostil generalizada } \\
\text { y violencia sin ira }\end{array}$ \\
\hline Distorsiones cognitivas & Nivel bajo & Atribuciones negativas & Justificación de la violencia \\
\hline Estilo de personalidad & Dependiente, pasivo-agresivo, evitativo & Límite & Antisocial y narcisista \\
\hline Riesgo & Bajo & Medio & Alto \\
\hline Recomendación terapéutica & $\begin{array}{c}\text { Programa breve centrado en la expre- } \\
\text { sión adecuada de las emociones y en } \\
\text { la gestión de la ira } \\
\text { Valorar la necesidad del tratamiento } \\
\text { toxicológico }\end{array}$ & $\begin{array}{l}\text { Programa medio/largo. Especial rele- } \\
\text { vancia al ámbito relacional (apego) y a } \\
\text { la psicopatología ansiosa o depresiva } \\
\text { Posible necesidad de tratamiento toxi- } \\
\text { cológico }\end{array}$ & $\begin{array}{l}\text { Programa largo. Muy estructurado, } \\
\text { directivo y centrado en las consecuen- } \\
\text { cias negativas de su conducta } \\
\text { Frecuente necesidad de tratamiento } \\
\text { toxicológico }\end{array}$ \\
\hline
\end{tabular}


Junto a los aspectos que comúnmente se abordan en las terapias con agresores (distorsiones, empatía, habilidades sociales, etc.), existen algunas variables específicas que pueden diferenciar a los agresores y que implican alterar las estructuras de los programas de intervención. Entre ellas, podemos señalar el abuso de drogas, el tipo de apego, la conducta antisocial/psicopatía y otros trastornos de la personalidad.

En la tabla 3 se describen de forma esquemática algunas de las características y recomendaciones terapéuticas para cada tipo de agresor.

Conviene recordar que, hasta la fecha, no existen estudios publicados que hayan puesto a prueba intervenciones basadas en las tipologías (Whitaker y Niolon, 2009) y que, además, en la Unión Europea se encuentran en fase de desarrollo las políticas para abordar esta problemática violenta (Graham-Kevan, 2007). Por otra parte, muchas de las intervenciones adoptadas obedecen a presiones de grupos políticos o ideológicos y no científicos. Todo ello hace que directrices como las presentadas en este estudio sean de vital importancia y de un elevado interés académico y social.

\section{Una experiencia piloto en el centro penitenciario Brians-2}

En 2008 se inició en el centro penitenciario Brians-2 (Barcelona) un proyecto cuyo objetivo era realizar una primera aproximación al estudio de las tipologías de agresores de pareja. Tras la obtención de los primeros resultados (Loinaz, 2009; Loinaz et al., 2010) y su posterior validación (Loinaz, 2010), se valoró la posibilidad de comenzar a aplicar dicha clasificación en el diseño de tratamientos diferenciados para cada tipo de agresor. A su vez, se pretendía hacer compatible el procedimiento (tanto en la evaluación como en el tratamiento) con los estándares internacionales y los requisitos de predicción de riesgo que se aplican en la actualidad en Cataluña (RISCANVI). Así pues, en 2009 se diseñaron los dos programas de tratamiento que figuran en las tablas 4 y 5 (partiendo del programa marco existente para todas las prisiones catalanas) y se asignó a los sujetos a una modalidad u otra siguiendo la metodología tipológica.

Ambas modalidades se componen de tres bloques: 1) Motivación para la terapia (responsabilidad, reconocimiento y empatía); 2) Intervención (a/ ciclo de la violencia, distorsiones cognitivas y control emocional; b/ autoestima, celos y estilo de vida saludable; y c/ asertividad y comunicación, resolución de problemas); y 3) Prevención de recaídas (identificación de situaciones de riesgo y estrategias adecuadas de afrontamiento). Estas modalidades se han desarrollado en un formato grupal (con la posibilidad de disponer de sesiones individuales de apoyo o seguimiento) y han incluido la evaluación pre y post de variables psicológicas, así como la valoración clínica del progreso en la terapia mediante un formulario específico adaptado de Gondolf y Wernik (2009) (ver Anexo 1).

El programa "corto" (tabla 4) ha consistido en 24 sesiones ( 2 sesiones semanales de 2 horas). En él se ha tratado con mayor intensidad el ámbito emocional: adquisición de habilidades comunicativas, asertividad, gestión adecuada de la ira, empatía con la víctima (junto al resto de los contenidos propios del programa). Los sujetos asignados a este programa son aquellos que por sus características corresponden al perfil normalizado ("violentos con la pareja/estables emocionalmente/integrados socialmente"), cuyo riesgo es menor y no presentan alteraciones psicopatológicas que dificulten la terapia (ni trastornos mentales, ni adicciones).

El programa "largo" (tabla 5) duplica su duración (48 sesiones) e incluye la evaluación de problemas toxicológicos y su relación con la violencia (pero no su tratamiento, del que se encarga un equipo específico de forma serial o paralela). Su contenido enfatiza el tratamiento de las distorsiones cognitivas (especialmente las mantenedoras de la conducta violenta), el control de la ira y la impulsividad, así como el cambio de las contingencias de la conducta violenta y la asunción de responsabilidades. A él han sido asignados aquellos sujetos con un perfil antisocial o patológico ("violentos generalizados/poco estables emocionalmente/no integrados socialmente"), que presentan mayor riesgo de conducta violenta y en los que su violencia está relacionada con problemas toxicológicos y hábitos delictivos. 
Tabla 4. Estructura del programa corto

Duración: 3 meses (24 sesiones)

2 sesiones semanales conjuntas de educador y psicólogo

DESTINATARIOS: agresores del grupo 1 (violentos con la parejalestables emocionalmente/integrados socialmente)

\section{OTRAS VARIABLES:}

- Baja severidad de la violencia (pese a que se pueden dar esporádicamente agresiones graves)

- Consumo de drogas similar al de la población general (posible abuso de alcohol)

- Represión emocional y mala gestión de la ira

- Estilo de apego seguro o preocupado

\begin{tabular}{|c|c|c|}
\hline BLOQUE & SESIONES & RESPONSABLE \\
\hline 1 & $\begin{array}{l}\text { 1- Presentación del programa, objetivos y compromiso terapéutico } \\
\text { 2- Responsabilidad, mecanismos de defensa } \\
\text { 3- Reconocimiento del problema (corto: "Amores que matan") } \\
\text { 4- Reconocimiento del problema } \\
\text { 5- Empatía y tipos de maltrato } \\
\text { 6- Empatía (carta a la víctima) }\end{array}$ & $\begin{array}{l}\text { ED, PSI, TS, JC } \\
\text { ED, PSI } \\
\text { ED, PSI } \\
\text { ED, PSI } \\
\text { ED, PSI } \\
\text { ED, PSI }\end{array}$ \\
\hline 2 & $\begin{array}{l}\text { 7- Explicación del ciclo de la violencia } \\
\text { 8- Vídeo "Te doy mis ojos" (resumen e identificación personal) } \\
\text { 9- Distorsiones cognitivas (teoría) } \\
\text { 10- Distorsiones cognitivas (confrontación de creencias) } \\
\text { 11- Control emocional. Ansiedad. Relajación } \\
\text { 12- Control emocional. Celos (reestructuración cognitiva) } \\
\text { 13- Control emocional. Ira y resentimiento 1 ( “) } \\
\text { 14- Control emocional. Ira y resentimiento } 2 \text { ( “) } \\
\text { 15- Autoestima y estilo de vida positivo } \\
\text { 16- Habilidades de comunicación (asertividad) } \\
\text { 17- Habilidades de comunicación en pareja } \\
\text { 18- Resolución de problemas 1. Detección y definición } \\
\text { 19- Resolución de problemas 2. Alternativas y consecuencias }\end{array}$ & $\begin{array}{l}\text { ED, PSI } \\
\text { ED, PSI } \\
\text { ED, PSI } \\
\text { ED, PSI } \\
\text { ED, PSI } \\
\text { ED, PSI } \\
\text { ED, PSI } \\
\text { ED, PSI } \\
\text { ED, PSI } \\
\text { ED, PSI } \\
\text { ED, PSI } \\
\text { ED, PSI } \\
\text { ED, PSI }\end{array}$ \\
\hline 3 & $\begin{array}{l}\text { 20- Recursos externos } \\
\text { 21- Cuestiones jurídicas (orden de protección de la víctima, responsabilidad civil y otras dudas) } \\
\text { 22- Identificación de situaciones de riesgo } \\
\text { 23- Estrategias de afrontamiento } \\
\text { 24- Cierre y valoraciones }\end{array}$ & $\begin{array}{l}\text { TS } \\
\text { JC } \\
\text { ED, PS } \\
\text { ED, PSI } \\
\text { ED, PSI }\end{array}$ \\
\hline
\end{tabular}

ED: educador; PSI: psicólogo; TS: trabajador social; JC: jurista criminólogo

El lector habrá podido comprobar cómo en ninguno de los dos programas se han incorporado aún sesiones específicas que aborden el estilo de apego, pese a que esta variable pueda ser tenida en cuenta de forma indirecta a través de las sesiones dedicadas a la autoestima, resolución de conflictos, asertividad y expresión emocional. Esta variable es aún novedosa y sus características e implicaciones para el tratamiento deben ser analizadas con mayor profundidad en futuras investigaciones.

Dado que ésta es una experiencia piloto todavía en curso, aún es necesario valorar los contenidos específicos desarrollados en cada programa, así como los resultados obtenidos en los mismos, para poder mejorar las técnicas con una base empírica y reformular los programas para mejorar su efectividad. Pese a esta limitación, se puede afirmar que la utilización de esta metodología tiene distintos beneficios. Por un lado, ayuda al terapeuta a gestionar de manera más segura los grupos, contando con participantes similares entre sí y conociendo las temáticas concretas sobre las que deberá incidir en cada grupo. Asimismo, puede ayudar a reducir la carga laboral al focalizar los esfuerzos en aquellos sujetos que más los requieren e invertir menos sesiones en los demás. Por último, homogeneizar los grupos facilita la eficiencia de la terapia, la evolución simultánea de los sujetos, la compatibilidad entre ellos y la anticipación del terapeuta respecto a lo que puede ocurrir en el grupo. 
Tabla 5. Estructura del programa largo

Duración: 6 meses (48 sesiones)

2 sesiones semanales

DESTINATARIOS: agresores del grupo 2 (violentos generalizados/poco estables emocionalmente/no integrados socialmente)

\section{OTRAS VARIABLES POSIBLES:}

- Severidad de la violencia media-alta

- Abuso de drogas/politoxicomanía (requiere evolución positiva en el programa de toxicomanías previo o paralelo)

- Impulsividad alta

- Mayor nivel de distorsiones cognitivas

- Estilo de apego rechazante

\begin{tabular}{lll} 
BLOQUE & SESIONES & R \\
\hline & 1- Presentación del programa, objetivos y compromiso terapéutico & ED \\
2- Responsabilidad y mecanismos de defensa & ED \\
3- Visionado del corto “Amores que matan" & PSI \\
4- Culpa frente a Responsabilidad & ED \\
5- Reconocimiento del problema1 & PSI \\
6- Reconocimiento del problema 2 & ED \\
7- Problemas con el alcohol y otras drogas; excusas & PSI \\
8- Empatía y tipos de maltrato & ED \\
9- Empatía (técnica: carta a la víctima) & PSI \\
10- Empatía (técnica: silla vacía) & PSI
\end{tabular}

11- Explicación del ciclo de la violencia $\quad$ ED

12- Discusión de estereotipos de género PSI

ED

14- Visionado de un vídeo y ejemplos gráficos $\quad$ ED

15- Distorsiones cognitivas (teoría) PSI

16- Inventario de distorsiones cognitivas sobre la mujer $\quad$ ED

17- Inventario de distorsiones cognitivas sobre la violencia

18- Visionado de la película "Te doy mis ojos" (escrito sobre identificación) ED

19- Debate sobre la película (discusión sobre identificación)

20- Mesa sobre el debate racional

21- Control emocional. Ansiedad. Exposición teórica

22- Entrenamiento en respiración $\quad$ ED

23- Entrenamiento en relajación muscular $\quad$ ED

24- Entrenamiento en relajación mental

2 25- Control emocional de los celos (reestructuración cognitiva) $\quad$ ED

27- Control emocional. Ira y resentimiento 2 (“) PSI

28- Pensamientos automáticos $\quad$ PSI

29- Detección de conductas violentas (ejercicio: autorregistros) PSI

30- Autoestima y autoconcepto $\quad$ ED

31- Estilo de vida positivo $\quad$ ED

32- Proyecto de vida personal PSI

33- Habilidades de comunicación (asertividad) $\quad$ ED

34- Habilidades de comunicación (dar / recibir quejas) $\quad$ PSI

35- Habilidades de comunicación (dar / recibir elogios) $\quad$ ED

36- Habilidades de comunicación en pareja

37- Mitos y falacias sobre las relacionales-sexuales $\quad$ ED

38- Resolución de problemas 1. Detección $\quad$ PSI

39- Resolución de problemas 2. Definición $\quad$ ED

40- Resolución de problemas 3. Alternativas y consecuencias $\quad$ PSI

41- Resolución de problemas 4. Caso práctico $\quad$ ED

42- Explicación de recursos externos $\quad$ TS

43- Explicación de cuestiones jurídicas (orden de protección de la víctima, responsabilidad civil y otras dudas) JC

44- Identificación de situaciones de riesgo PSI

45- Estrategias de afrontamiento $\quad$ ED

46- Análisis del delito $\quad$ PSI

47- Plan individual de prevención $\quad$ ED

48- Cierre y valoraciones $\quad$ ED, PSI

* Las sesiones han podido desarrollarse de forma conjunta por el psicólogo y el educador según la conveniencia.

ED: educador; PSI: psicólogo; TS: trabajador social; JC: jurista criminólogo 
Desde una perspectiva clínica, esta aproximación tipológica puede ser la principal alternativa de elección a la hora de diseñar programas lo más ajustados a las necesidades reales de cada sujeto, especialmente en contextos en los que los recursos no permitan un tratamiento individualizado. El terapeuta conocerá de antemano la problemática de cada individuo mediante el protocolo de evaluación; a su vez, las técnicas empleadas en la terapia podrán dar respuesta a dichas problemáticas compartidas entre cada subtipo de agresores.

\section{Conclusiones y futuras líneas de investigación}

En resumen, el tratamiento de los agresores debe ser considerado uno de los principales frentes de actuación a la hora de prevenir la violencia contra la pareja, especialmente en aquellos sujetos que ya han sido condenados por una agresión. Distintos estudios han propuesto que la ineficacia de algunas intervenciones se debe, en parte, a no prestar atención a las necesidades terapéuticas específicas de los agresores y a utilizar únicamente programas homogéneos y estandarizados. Como se ha señalado, el estudio de las tipologías y de los perfiles diferenciales ha abierto un nuevo terreno de actuación desde el que se pueden diseñar programas ajustados a cada tipo de agresor y evitar así, al menos parcialmente, las elevadas cifras de reincidencia y abandono de los tratamientos.

Esta perspectiva de intervención con agresores de pareja aún debe ser estudiada con mayor profundidad. De este modo, los estudios longitudinales y de eficacia terapéutica realizados en nuestro entorno (ver Echeburúa et al., 2009) deben ser replicados en distintos ámbitos, como las prisiones.

Respecto a las líneas de actuación futura, se considera de vital importancia extender el estudio de las tipologías y de los perfiles diferenciales a los distintos espacios de tratamiento con agresores. Asimismo, deben ser valorados empíricamente los programas basados en las tipologías que se estén desarrollando y descritas de forma detallada aquellas intervenciones específicas de las que se pueden beneficiar los distintos tipos de agresores. Por último, puede resultar de interés adaptar la metodología tipológica a la clasificación de los agresores partiendo de su riesgo (evaluado mediante herramientas como el RISCANVI, de aplicación actual en prisiones de Cataluña). Ello puede permitir tomar como referencia el riesgo de reincidencia del agresor y gestionar, mediante las tipologías, las necesidades terapéuticas y los recursos disponibles.

No debemos olvidar que el fin último debe ser garantizar la máxima seguridad de las víctimas, modificar las conductas agresivas de los sujetos -sea cual sea su origen- y evitar futuras agresiones y nuevas victimizaciones, todo ello mediante las actuaciones más adecuadas.

\section{Referencias}

Amor, P.J., Echeburúa, E. y Loinaz, I. (2009). ¿Se puede establecer una clasificación tipológica de los hombres violentos contra su pareja? International Journal of Clinical and Health Psychology, 9, 519-539.

Bowen, E., Gilchrist, E. y Beech, A.R. (2008). Change in treatment has no relationship with subsequent reoffending in U.K. domestic violence sample: a preliminary study. International Journal of Offender Therapy and Comparative Criminology, 52, 598-614.

Cavanaugh, M.M. y Gelles, R. J. (2005). The utility of male domestic violence offender typologies: New directions for research, policy, and practice. Journal of Interpersonal Violence, 20, 155-166.

Daly, J.E. y Pelowski, S. (2000). Predictors of dropout among men who batter: A review of studies with implications for research and practice. Violence and Victims, 15, 137-160.

Dutton, D.G. (2007). The abusive personality. Violence and control in intimate relationships (2 $\left.2^{\mathrm{a}} \mathrm{ed}.\right)$. New York: The Guilford Press.

Dutton, D.G. (2008). Blended behavior therapy for intimate violence. En A.C. Baldry y F.W. Winkel (Eds.), 
Intimate partner violence prevention and intervention: The risk assessment and management approach (pp. 133-146). New York: Nova Science Publishers.

Dutton, D.G. y Sonkin, D.J. (Eds.) (2003). Intimate violence: Contemporary treatment innovations. Binghamton, NY: Haworth Press.

Dutton, D.G., Bodnarchuk, M., Kropp, R., Hart, S.D. y Ogloff, J.P. (1997). Client personality disorders affecting wife assault post-treatment recidivism. Violence and Victims, 12, 37-50.

Echeburúa, E. (2004). Tratamiento del agresor doméstico. En J. Sanmartín (Coord.), El laberinto de la violencia, (pp. 293-307). Barcelona: Ariel.

Echeburúa, E. y Corral, P. (1998). Manual de violencia familiar. Madrid: Siglo XXI.

Echeburúa, E. y Fernández-Montalvo, J. (2007). Male batterers with and without psychopathy: an exploratory study in Spanish prisons. International Journal of Offender Therapy and Comparative Criminology, 51, 254263

Echeburúa, E., Corral, P., Fernández-Montalvo, J. y Amor, P.J. (2004). ¿Se puede y debe tratar psicológicamente a los hombres violentos contra la pareja? Papeles del Psicólogo, 88, 20-28.

Echeburúa, E., Sarasua, B., Zubizarreta, I. y Corral, P. (2009). Evaluación de la eficacia de un tratamiento cognitivo-conductual para hombres violentos contra la pareja en un marco comunitario: una experiencia de 10 años (1997-2007). International Journal of Clinical and Health Psychology, 9, 199-217.

Fernández-Montalvo, J. y Echeburúa, E. (2008). Trastornos de la personalidad y psicopatía en hombres condenados por violencia grave contra la pareja. Psicothema, 20, 193-198.

Fernández-Montalvo, J., Echeburúa, E. y Amor, P. J. (2005). Aggressors against women in prison and in community: an exploratory study of a differential profile. International Journal of Offender Therapy and Comparative Criminology, 49, 158-167.

Gondolf, E.W. (2000). A 30-month follow-up of court-referred batterers in four cities. International Journal of Offender Therapy and Comparative Criminology, 44, 111-128.

Gondolf, E.W. y White, R.J. (2001). Batterer program participants who repeatedly reassault: Psychopathic tendencies and other disorders. Journal of Interpersonal Violence, 16, 361-380.

Gondolf, E.W. y Wernik, H. (2009). Clinician ratings of batterer treatment behaviors in predicting reassault. Journal of Interpersonal Violence, 24, 1792-1815.

Graham-Kevan, N. (2007). Domestic Violence: Research and Implications for Batterer Programmes in Europe. European Journal on Criminal Policy and Research, 13, 213-225.

Johnson, S.A. (2007). Physical abusers and sexual offenders: Forensic and clinical strategies (pp. 261-283). Boca Raton, FL: CRC/Taylor \& Francis.

Klein, A.R. y Tobin, T. (2008). A longitudinal study of arrested batterers, 1995-2005. Career criminals. Violence Against Women, 14, 132-157.

Loinaz, I. (2009). Aproximación teórica y empírica al estudio de las tipologías de agresores de pareja: análisis descriptivo de variables e instrumentos de evaluación en el centro penitenciario Brians-2. Madrid: Ministerio del Interior, Secretaría General Técnica.

Loinaz, I. (2010). Estudio de las tipologías de agresores de pareja en centros penitenciarios. Estudio validativo. Ayudas a la investigación 2009. Barcelona: Centro de Estudios Jurídicos y Formación Especializada.

Loinaz, I., Echeburúa, E. y Torrubia, R. (2010). Tipología de agresores contra la pareja en prisión. Psicothema, $22,106-111$.

Loinaz, I., Ortiz-Tallo, M., Sánchez, L.M. y Ferragut, M. (2011). Clasificación multiaxial de agresores de pareja en dos centros penitenciarios. International Journal of Clinical and Health Psychology, 11, en prensa.

Loinaz, I., Torrubia, R., Echeburúa, E., Navarro, J.C. y Fernández, L. (2009). Implicaciones de las tipologías de agresores de pareja para el tratamiento en prisión. Cuadernos de Medicina Psicosomática y Psiquiatría de Enlace, 91, 19-25.

Redondo, S. (2008). Manual para el tratamiento psicológico de los delincuentes. Madrid: Pirámide. 
Russell, M.N. (2002). Changing beliefs of spouse abusers. En J. McGuire (Ed.), Offender rehabilitation and treatment: Effective programmes and policies to reduce re-offending (pp.243-258). Chichester: John Wiley \& Sons.

Stith, S.M., Rosen, K.H. y McCollum, E.E. (2003). Effectiveness of couples treatment for spouse abuse. Journal of Marital and Family Therapy, 29, 407-426.

Stith, S.M., Rosen, K.H., McCollum, E.E. y Thomsen, C.J. (2004). Treating intimate partner violence within intact couple relationships: outcomes of multi-couple versus individual couple therapy. Journal of Marital and Family Therapy, 30, 305-318.

Whitaker, D.J. y Niolon, P.H. (2009). Advancing interventions for perpetrators of physical partner violence: Batterer intervention programs and beyond. En D.J. Whitaker y J.R. Lutzker (Eds.), Preventing partner violence. Research and evidence-based intervention strategies (pp. 169-192). Washington, DC: American Psychological Association.

Manuscrito recibido: $17 / 05 / / 2010$

Revisión recibida: 21/06/2010

Manuscrito aceptado: 25/06/2010

Anexo 1. Valoración clínica de la evolución terapéutica (adaptado de Gondolf y Wernik, 2009)

Valórese cada criterio en una escala de 0 a 5 basándose en la impresión personal y las observaciones realizadas durante el programa de tratamiento.

\begin{tabular}{|lll|}
\hline $5=$ absolutamente presente & $4=$ muy presente & $3=$ algo presente \\
$2=$ un poco presente & $1=$ muy poco presente & $0=$ no aplicable \\
\hline
\end{tabular}

asistencia: asiste con puntualidad a las sesiones; socializa tras la sesión; informa sobre ausencias o tiene excusa justificada.

__ no-violencia: no ha agredido recientemente a la pareja, hijos u otras personas; sin aparentes amenazas, intimidaciones o manipulaciones.

sobriedad: acude a las sesiones sobrio; sin aparente consumo de sustancias entre sesiones; cumple con los programas propuestos para consumo de sustancias.

__ aceptación: admite la existencia de violencia y abusos; no minimiza, ni culpabiliza (a la víctima o a otros), ni se excusa; se da cuenta de su responsabilidad.

utilización de técnicas: toma medidas de forma consciente para evitar la violencia o la ira; refiere el uso de técnicas aprendidas en el programa (como tiempo- fuera, habilidades de resolución de conflictos, etc.); realiza los ejercicios propuestos.

búsqueda de ayuda: busca información sobre distintas alternativas; comparte opiniones con otros miembros del grupo; recurre a la ayuda de otros compañeros de grupo; está abierto a recibir apoyo en el futuro.

conciencia del proceso: permite a los demás hablar en su momento; acepta las contribuciones de los demás; hace preguntas a los demás sin interrogar.

implicación activa: muestra un lenguaje corporal y respuesta no verbal de atención; mantiene contacto visual; habla con sentimiento; sigue el tema de debate en los comentarios.

apertura personal: muestra sus conflictos, sentimientos, miedos y dudas personales; no estanca o evita los problemas; no es sarcástico ni defensivo.

lenguaje sensible: manifiesta respeto hacia la pareja y las mujeres en general; no utiliza un lenguaje sexista ni jerga peyorativa; con-

TOTAL trola que otros no utilicen lenguaje sexista. 